\title{
Influence of a Point-to-Plane DC Negative Corona Discharge on Gel Surfaces
}

\author{
Y. Klenko, V. Scholtz
}

Point-to-plane corona discharge is widely used for modifying polymer surfaces for biomedical applications and for sterilization and decontamination. This paper focuses on an experimental investigation of the influence of the single-point and multi-point corona discharge electric field on gel surface. .Three types of gelatinous agar were used as the gel medium: blood agar, nutrient agar and Endo agar. The gel surface modification was studied for various time periods and discharge currents.

Keywords: Point-to-plane corona, electrohydrodynamic deformation, gel ion-conducting electrode.

\section{Introduction}

Non-equilibrium atmospheric pressure discharges have recently been used in emerging novel applications such as the surface modification of polymers, the absorption or reflection of electromagnetic waves, biomedical treatments and plasma-aided combustion [1]. Among these various interesting new applications, the interaction of atmospheric pressure "cold" plasmas with a biological medium promises to open new horizons in the medical and environmental fields.

The main methods of sterilization, i.e., inactivation of living microorganisms, are based on thermal treatment (dry or moist heat), chemical treatment (e.g., EtO, $\mathrm{H}_{2} \mathrm{O}_{2}$ ) or exposure to ionizing radiation (X-ray, gamma radiation, e-beams). These methods have specific drawbacks linked to their conditions of operation, e.g., the toxicity of the active agent used, the temperature conditions, or the size of the installation. Clearly, plasma sources have some advantages such as low temperature (at or near room temperature), no risk of arcing, operation at atmospheric pressure, portability, optional hand-held operation, etc. Therefore low-temperature non-equilibrium plasmas are playing an increasing role in biomedical applications, and reliable, user-friendly sources need to be developed. A point-to-plane corona discharge at atmospheric pressure is one of the atmospheric pressure discharges applicable for studying the interactions of atmospheric ions with microorganisms such as bacteria and fungi [2-4]. The bactericidal or fungicidal effect of corona discharge can be studied directly on a gel medium (agar), which is a gelatinous substance chiefly used as a culture medium for microbiological work. This technology minimizes the risk of accidental contamination of the samples, and makes the experiments relatively simple.

Corona discharges are usually realized in such a way that one of the electrodes has a small radius of curvature. This makes the electric field intensity very high close to this electrode in comparison to other points in the gap. Electrons and positive ions are egnerated. If the gas contains an electronegative component, electrons can attach to it to produce negative ions. Afterwards the ions drift to the corresponding electrode according to particle polarity. Their movement is broken by collisions with neutral atoms and molecules. This process can be classified as friction. Ions drifting to an elec- trode with a low radius of curvature (further referred to as a plate electrode) can also make the neutral gas move. This important phenomenon is called electric wind [5]. Due to ionic wind the gel surface is deformed, which results in a change in the corona discharge geometry. The discharge current and voltage change during the experiments in dependence on the surface deformation and the gel medium.

\section{Experimental part}

Fig. 1 shows the scheme of the apparatus used for generating a point-to-plane negative corona discharge at atmospheric pressure.

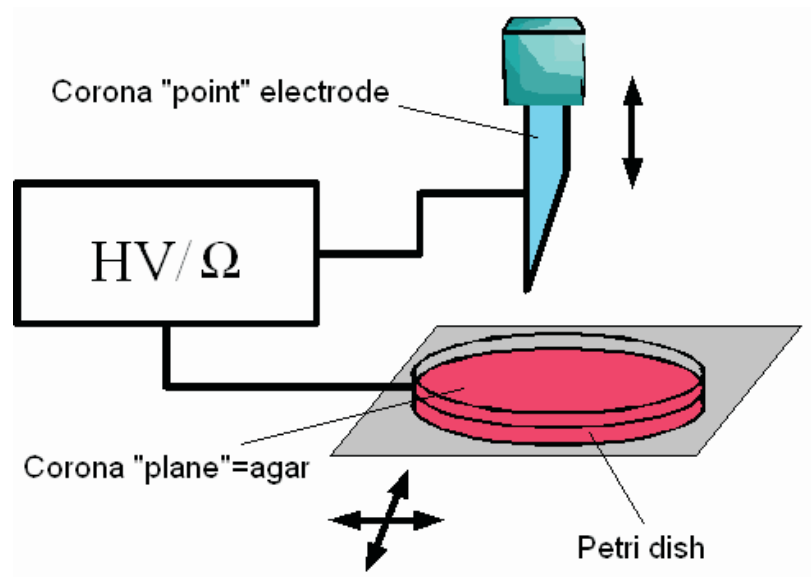

Fig. 1: Schematic of an open-air corona exposure single-point set-up with a Petri dish $90 \mathrm{~mm}$ in diameter

Each type of gel electrode was placed in a standard Petri dish (90 $\mathrm{mm}$ in diameter). The size of the gel layer electrode varied in the analyzed samples, the mean value being $8 \mathrm{~mm}$. The following gel types were used: blood agar, nutrient agar, Endo agar. All were prepared from defined fabric substrates. Six samples of each type of agar were used; the dates of sample preparation and storage of the samples in a cooling box varied. The point corona electrode was realized by an intramuscular medicinal hollow needle with an outer diameter of $0.7 \mathrm{~mm}$. The needle had a sharpened angle of 15 degrees. The position of the hollow needle was adjusted by a micro- 
metric screw placed inside the needle holder. The position of the Petri dishes could also be changed in two directions perpendicular to the needle movement and also perpendicular to each other. The position change of the hollow needle and the Petri dish was measured with precision $0.01 \mathrm{~mm}$.

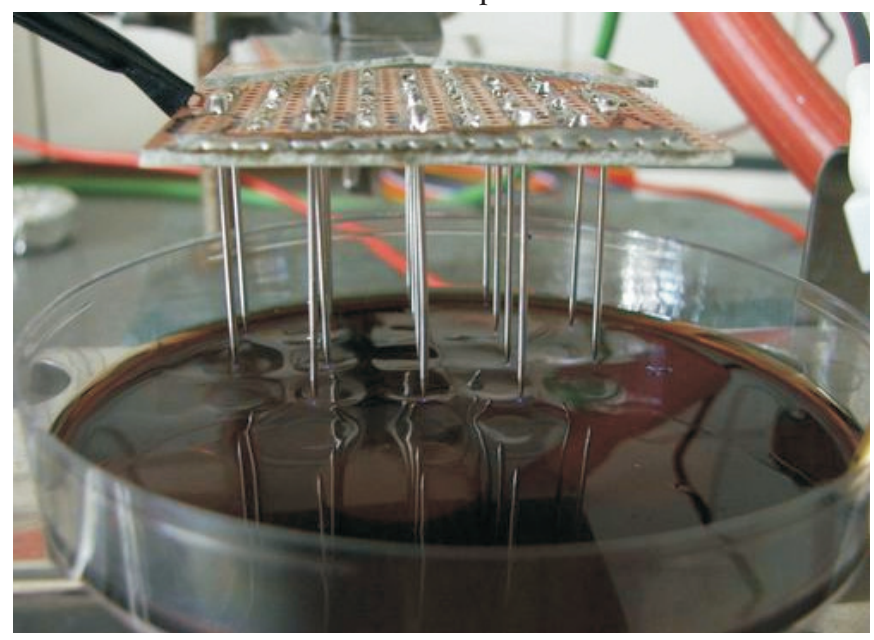

Fig. 2: Photograph illustrating the experimental set-up

When the needle was used as an electrode in the discharge regime, it was connected to a high voltage supply. The same needle was used detecting deformation in the measurement regime. The conductive connection between the tip of the hollow needle and the gel surface was checked using an ohmmeter. Initially, the tip of the hollow needle was placed $6 \mathrm{~mm}$ above the gel surface and the positions of the needle and the high voltage source set-up remained unchanged during corona treatment. In the deformation measurements the dimple depth was measured as the change in the distance between the electrodes. The needle was moved perpendicularly toward the gel surface. the contact between the needle tip and the gel surface was detected by the ohmmeter. The exposition times varied (1, 2, 4, 8, $16 \mathrm{~min})$.

\section{Results}

Fig. 3 is a photograph of the gel surface deformations caused by the corona discharge. A matrix multi-point-to-plane corona discharge was used in this case.

Fig. 3 shows the gel deformation due to the multi system point-to-plane corona discharges. Due to their simplicity, the measurements were made with a single-needle-to-gel electrode system.

Fig. 4. shows the dependence of dimple depth on the gel surface exposition in a point-to-plane corona discharge. During the measurements, the corona current decreased from initial value $(50 \mu \mathrm{A})$ according to the gel electrode deformation.

The dependence of dimple depth on point-to-plane corona initial current is show in Fig. 5. The duration of the gel surface exposition in the corona discharge was 16 minutes.

The diameter of the dimple at $0.3 \mathrm{~mm}$ depth was taken as a standard characteristic dimension of the area affected by the point-to-plane corona discharge. After the influence of the corona, the needle was moved $6.3 \mathrm{~mm}$ towards the gel surface, and standard diameter d was measured. Fig. 6 presents

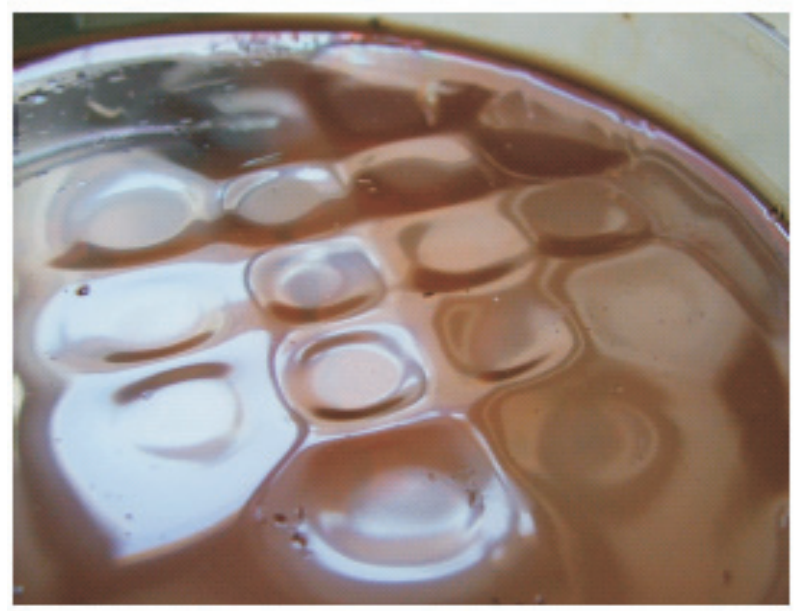

Fig. 3: Photograph of a Petri dish showing the gel surface deformation caused by the corona discharge exposition

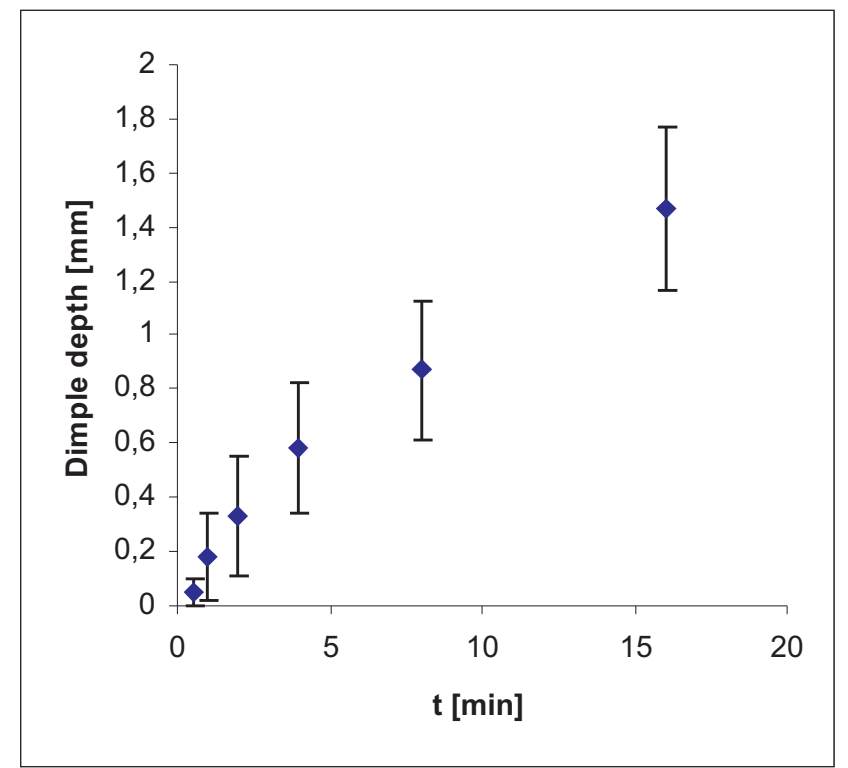

Fig. 4: Dependence of dimple depth on exposition time at discharge current $50 \mu \mathrm{A}$

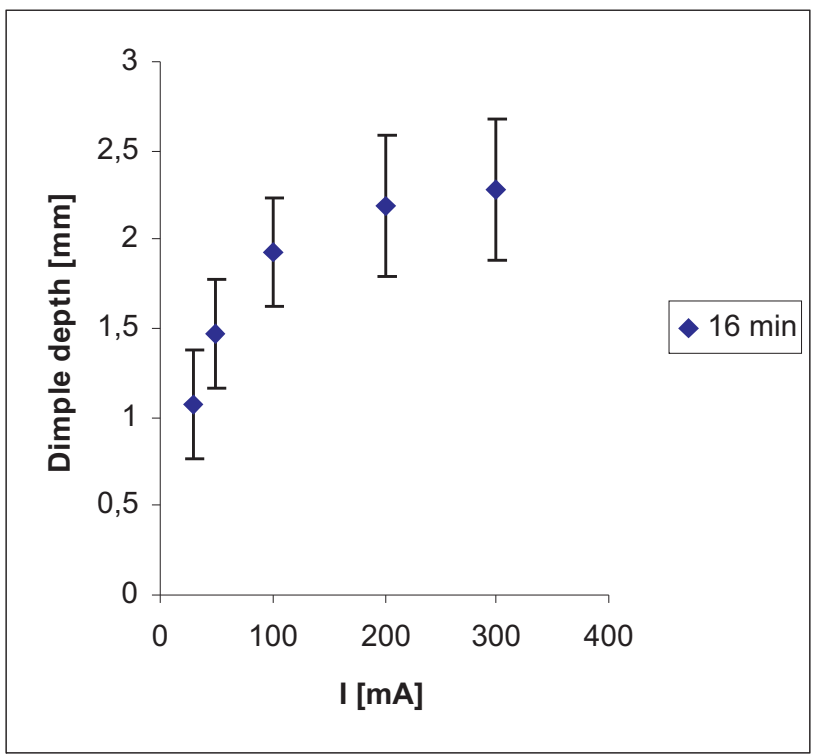

Fig. 5: Dependence of dimple depth on discharge current (exposition time $16 \mathrm{~min}$ ) 


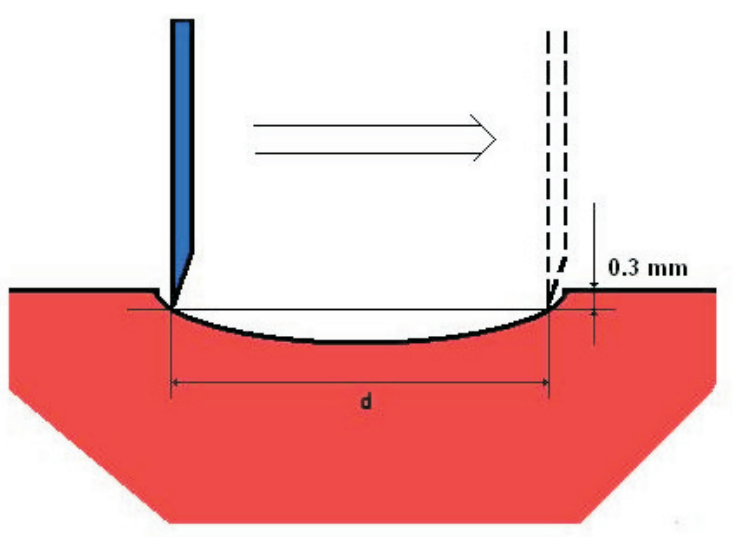

Fig. 6: The process of measuring standard diameter $d$

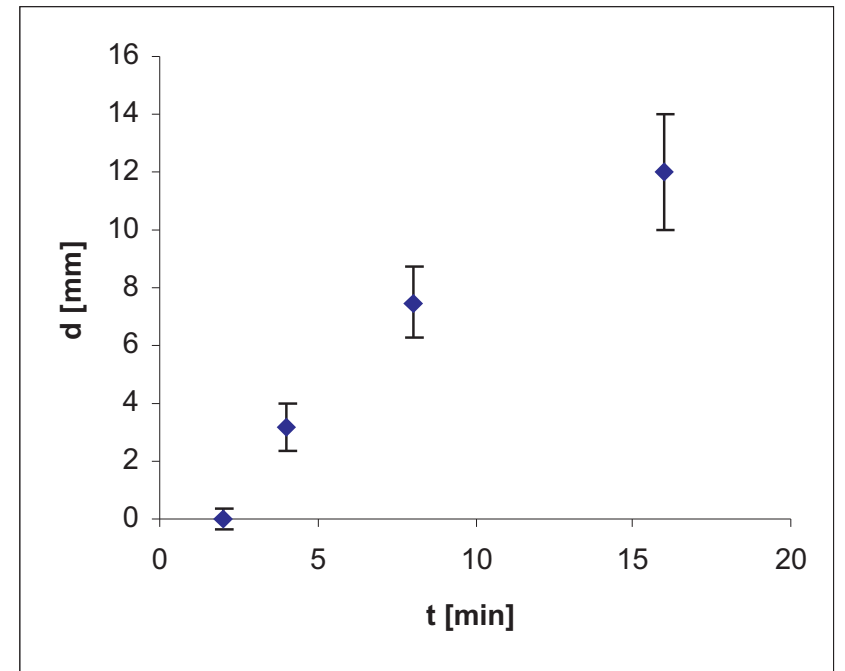

Fig. 7: Standard diameter increment with an increase in discharge current (influence time $16 \mathrm{~min}$ )

the process of measuring the standard diameter. The dependence of the standard diameter on exposition duration is shown in Fig. 7. The initial corona current was $50 \mu \mathrm{A}$.

The dependence of standard diameter $\mathrm{d}$ on the initial corona current is shown in Fig. 8. The exposition duration was 16 minutes.

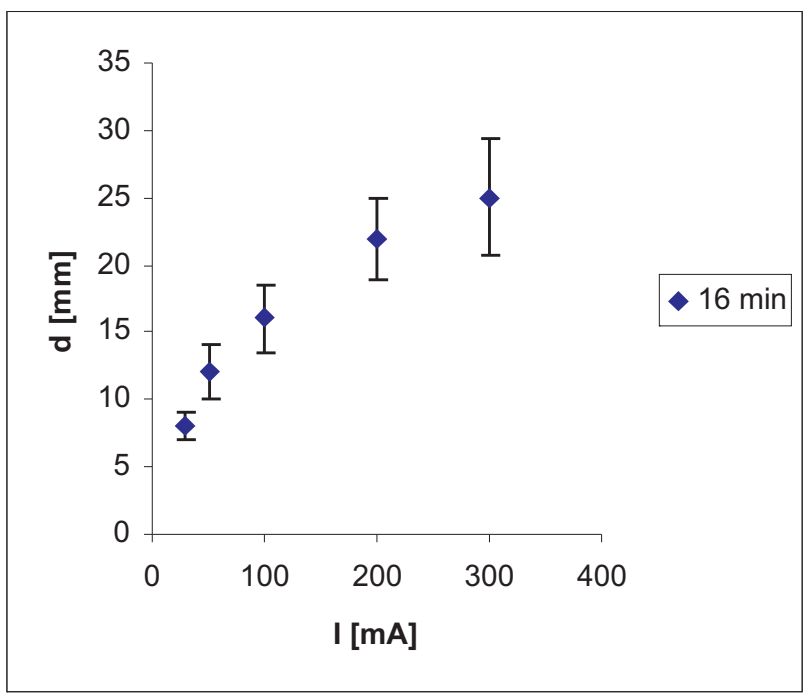

Fig. 8: Standard diameter dependence of treatment time (discharge current $50 \mu \mathrm{A}$ )

\section{Conclusion}

We developed an apparatus that generates a DC negative point-to-plane corona discharge of various parameters in air at atmospheric pressure. In addition, we attempted to develop a method enabling a quantitative evaluation of the action the discharge on an artificial bacterial culture. We also studied the influence of the electric field of the corona discharge on the substrate formed by the agar gel.

The electrohydrodynamic deformation of the substrate was observed. The gel surface was deformed by the ionic wind in the point-to-plane corona. The deformation of the gel surface was comparable to the distances between the electrodes used in sterilization experiments [2, 3]. The electrohydrodynamic deformation effect redistributed the corona current during gel exposition to a corona. Moreover Figs. 4-7 show that the gel surface deformation cannot be predicted for a specific gel type (although a relatively precise method of gel surface position determination was used). The variation of the deformation characteristic dimensions may be explained by the fact that the water content in the used agar plates used here changed during storage, and consequently the gel electrode viscosity also changed.

\section{Acknowledgments}

The research presented in this paper was supervised by Doc. J. Píchal, MUDr. Ing. V. Kříha and Prof. L. Aubrecht, FEE CTU in Prague, and has been supported by GAČR grant No. 202-03-H162 "Advanced Study in Physics and Chemistry of the Plasma".

\section{References}

[1] Laroussi, M., Tendero, C., Lu, X., Alla, S., Hynes, W. L.: Inactivation of Bacteria by the Plasma Pencil. Plasma Process Polym. 2006, 3, p. 470-473.

[2] Scholtz, V., et al.: Corona Discharge: a Simple Method of its Generation and Study of its Bacterial Properties. Cz.J. Phys., Vol. 56 (2006), Suppl. B, p. 1333-1338.

[3] Scholtz, V., et al.: The Study of Bactericidal Effects of Corona Discharge at Atmospheric Pressure. In Proceedings of the $33^{\text {rd }}$ EPS Conference on Plasma Phys. Rome (Italy), 2006, Vol. 301, p. 4.007.

[4] Sigmond, R. S., Kurdelova, B., Kurdel, M.: Action of Corona Discharges on Bacteria and Spores. Cz. J. Phys., Vol. 49 (1999), No. 3, p. 405-420.

[5] Kurdel, M., Morvova, M.: DC Corona Discharge Influence on Chemical Composition in Mixtures of Natural Gas with Air and is Combustion Exhaust with Air. Cz. J. Phys., Vol. 47 (1997), No. 2, p. 205-215.

\footnotetext{
Yuliya Klenko

e-mail: klenkj1@feld.cvut.cz

Vladimir Scholtz

e-mail:vscholtz@gmail.com

Czech Technical University

Faculty of Electrical Engineering

Technická 2

16627 Praha, Czech Republic
} 